\title{
Recent trends in physician diagnosed COPD in women and men in the UK
} Joan B Soriano, William C Maier, Peter Egger, George Visick, Bharat Thakrar,
Jennie Sykes, Neil B Pride
Department of Worldwide

Epidemiology, GlaxoWellcome Research and Development, Middlesex, UK J B Soriano

W C Maier

P Egger

G Visick

Department of Medical Data Sciences B Thakrar

Department of Respiratory Clinical Development

J Sykes

Respiratory and Environmental Health Research Unit, IMIM, Barcelona, Spain

J B Soriano

National Heart and Lung Institute, Imperial College School of Medicine, London, UK N B Pride

Correspondence to: Dr J B Soriano, Department of Worldwide Epidemiology, GlaxoWellcome R\&D, Greenford, Middlesex UB6 0HE, UK email: jbs42397@glaxowellcome. co.uk

Received 28 October 1999 Returned to authors 10 February 2000 Revised version received 19 April 2000 Accepted for publication 2 June 2000

\begin{abstract}
Background-Recent trends in physician diagnosed chronic obstructive pulmonary disease (COPD) in the UK were estimated, with a particular focus on women. Methods-A retrospective cohort of British patients with COPD was constructed from the General Practice Research Database (GPRD), a large automated database of UK general practice data. Prevalence and all-cause mortality rates by sex, calendar year, and severity of COPD, based on treatment only, were estimated from January 1990 to December 1997.

Results-A total of 50714 incident COPD patients were studied, $23277(45.9 \%)$ of whom were women. From 1990 to 1997 the annual prevalence rates of physician diagnosed COPD in women rose continuously from $0.80 \%(95 \%$ CI 0.75 to 0.83$)$ to $1.36 \%$ $(95 \%$ CI 1.34 to 1.39$)$, (p for trend $<0.01$ ), rising to the rate observed in men in 1990. Increases in the prevalence of COPD were observed in women of all ages; in contrast, a plateau was observed in the prevalence of COPD in men from the mid 1990s. Allcause mortality rates were higher in men than in women (106.8 versus 82.2 per 1000 person-years), with a consistently increased relative risk in men of 1.3 even after controlling for the severity of COPD. Significantly increased mortality rates were also observed in adults aged less than 65 years. The mean age at death was 76.5 years; patients with severe COPD died an average of three years before those with mild disease $(p<0.01)$ and four years before the age and sex matched reference population.

Conclusions-While prevalence rates of COPD in the UK seem to have peaked in men, they are continuing to rise in women. This trend, together with the ageing of the population and the long term cumulative effect of pack-years of smoking in women, is likely to increase the present burden of COPD in the UK.

(Thorax 2000;55:789-794)
\end{abstract}

Keywords: chronic obstructive pulmonary disease; epidemiology; sex differences; prevalence; mortality

Chronic obstructive pulmonary disease (COPD) is a chronic disorder responsible for a major burden in health care. The World Health Report of 1998 states that 2.9 million adults die each year of COPD, and it ranks COPD as the fifth cause of mortality worldwide (just after ischaemic heart disease, cerebrovascular disease, acute lower respiratory infection, and tuberculosis) and as the fifth most prevalent disease (after iron deficiency anaemia, neck and back disorders, goitre and hypertensive disease) for $1997 .{ }^{1}$ Prevalence and mortality figures are expected to increase early in the $21 \mathrm{st}$ century, particularly in developing countries such as China, ${ }^{2}$ but the burden of COPD in developed countries is expected to remain substantial. The natural history of COPD in the community is largely unknown, particularly in women. Few mortality studies and use of health services studies have been performed to date in women. Most of the published reports of COPD that use an automated database come from a single source, the Saskatchewan study, ${ }^{3}$ in Canada. Further, one of the largest cohorts of COPD patients identified is the Barcelona cohort in Spain. ${ }^{4}$ Both databases have been proved to be valid tools, but they may not represent the UK experience and they have a relatively small number of female patients. In the UK, while the full effects of the male smoking epidemic have been seen, the full effects in women are not yet apparent. Mortality from lung cancer peaked in men in 1975 in the UK and has consistently declined since, while a steady increase is still occurring in women. ${ }^{5}$ Similar trends may be observed in COPD

In our study we characterised the descriptive epidemiology and natural history of COPD in the UK, with a particular focus on women, by studying a retrospective cohort of COPD patients in a large automated database, the General Practice Research Database (GPRD), with the aim of improving the understanding of recent trends in the prevalence and mortality rates of COPD in the UK.

\section{Methods}

The research protocol and manuscript were approved by the Scientific and Ethics Advisory Group of the GPRD.

\section{DATABASE}

The GPRD has been described in detail elsewhere ${ }^{67}$ Briefly, it was originally set up in May 1987 with the aim of recruiting sufficient practices to build up a database containing continuing information on four million patients in England and Wales. As of 1998, 525 practices have participated with a total population of 3.4 million patients - that is, $6.4 \%$ of the total population of England and Walesand 105 practices have been supplying data for four years or more. Several studies have shown 
that the study base is a representative sample of the general population, with an almost identical sex and age structure to the one provided by the Office of Population Census and Statistics. GPs from the participating surgeries are invited to enter all significant morbidity events on each individual patient in the computer record, irrespective of whether the event occurred in the surgery, at a visit, or over the telephone. All diagnoses and procedures communicated to the GP as a result of a hospital or other specialist visit (inpatient, outpatient, or accident and emergency unit) must be recorded when the GP is informed. The diagnosis, symptoms, procedure or investigation, referrals, and their outcome must be entered into the relevant sections of the medical record. When a patient dies the date of death is recorded in the computerised patient medical records.

IDENTIFICATION OF COPD CASES, COPD SEVERITY, AND DEATH

The GPRD system uses the OXMIS coding system which is loosely based on the ICD-9 coding system: the first three digits of the OXMIS number corresponds, in most cases, to the first three digits of the ICD-9 codes. Physician diagnosed COPD was defined as any individual labelled with one or more of the OXMIS codes compatible with COPD. Validation studies of the GPRD documented the recording of medical data in the GP's computer to be nearly complete, and demonstrated good agreement between the recorded diagnosis and the diagnosis of the written clinical records. ${ }^{8} \mathrm{~A}$ recent paper comparing the GPRD with the 4th Morbidity Survey in General Practice reported an overall agreement in age-specific and sex-specific population rates of 11 respiratory conditions, including COPD. ${ }^{9}$ International guidelines categorise COPD as mild, moderate, or severe according to arbitrary values of percentage predicted forced expiratory volume in one second $\left(\mathrm{FEV}_{1}\right)$, but different thresholds have been proposed. ${ }^{10}$ Because $\mathrm{FEV}_{1}$ or other lung function data are usually not available in primary care and they are, indeed, not systematically recorded in the GPRD system, we assessed the severity of COPD according to recorded drug use. We considered all COPD patients when initially identified (one or more of the OXMIS codes compatible with COPD) as having mild COPD. Patients on regular treatment (defined as at least two prescriptions of the same drug group within six months) with inhaled/oral bronchodilators, xanthines, cromones, steroids, or combinations were considered as having moderate COPD from the time of the second prescription. Finally, COPD patients were considered severe when starting with oxygen or nebulised therapy. Severity could only be maintained or worsened with time, from mild to moderate to severe, as soon as treatment was increased. Individuals with a history of physician diagnosed COPD who moved from one practice not in the GPRD system to a GPRD practice were considered prevalent cases and were not counted as incident cases. In the current analysis the severity of COPD was assessed only in incident COPD cases from 1 January 1990.

As a result of the run in period of the GPRD database before 1990, we report prevalence data in all physician diagnosed COPD individuals (reported from 1990 or even historically before 1990), but incidence, mortality, and severity in newly physician diagnosed cases of COPD from 1990 onwards. Two further clarifications are necessary: patients diagnosed with COPD in GP practices other than the current one before 1990 were considered prevalent if their record was in the GPRD system and, by definition, all patients start with mild COPD and eventually move according to the algorithm to moderate or severe, even within days of the diagnosis of mild disease, as moderate COPD is considered from the time of a second prescription. Although some had regular treatment with a respiratory drug before having a first diagnosis of COPD, we found no individuals who were on nebulised or oxygen treatment before being diagnosed with COPD.

Validation of our COPD definitions was performed as follows: 100 computer database profiles were presented in a blind fashion to two independent observers (NBP and JBS) who labelled participants as having COPD or not and graded the severity of COPD by performing a comprehensive review of all database information. Outcomes of the previously described definitions of diagnosis of COPD and of the severity of COPD were compared with both observers, and agreement was quantified by the proportion of simple agreement and the Kappa index, an estimator that takes into account agreement that occurs by chance. Agreement between the diagnosis of COPD and the diagnosis by the two independent observers was $88 \%$ and $95 \%$ and produced kappa estimates of 0.76 and 0.90 , respectively. Interobserver agreement kappa was 0.78 (95\% CI 0.59 to 0.97$)$. There was also good agreement for the suggested COPD severity grading, quantified as a proportion of agreement of $58 \%$ and $79 \%$ and kappa estimates of 0.38 and 0.64, respectively; interobserver agreement kappa was 0.43 (95\% CI 0.22 to 0.64 ) for the severity of COPD classification.

Death was identified by the specific OXMIS code for death in the registration status file of the GPRD plus an extended set of 10 OXMIS codes from the medical file including the terms "death", "died", or "dead" (all programming and codes are available from the authors).

STATISTICAL ANALYSES

A total of 78172 patients with COPD were identified. The participants had about 5.9 million medical events and approximately 17.1 million treatment records. A sample size calculation was performed a priori: given a cohort of 50000 COPD patients with differing severity, and assuming an all-cause mortality rate per year at a frequency of 20 per 1000 person-years (a very conservative estimate), we had more than $95 \%$ power to detect a relative risk of 1.2 or larger compared with a general population sample or within COPD severity groups with a 
Table 1 Characteristics of COPD patients and population controls by sex

\begin{tabular}{|c|c|c|c|c|c|}
\hline & \multicolumn{4}{|l|}{$C O P D$} & \multirow[t]{2}{*}{ Control } \\
\hline & All & Mild & Moderate & Severe & \\
\hline \multicolumn{6}{|l|}{ Women } \\
\hline No. & 23277 & 7939 & 13440 & 1898 & 23259 \\
\hline Mean (SD) age at diagnosis & $66.7(15.5)$ & $65.8(18.6)$ & $66.9(13.8)$ & $68.8(11.9)$ & None \\
\hline Total person-years & 67913 & 26924 & 38008 & 2981 & 107126 \\
\hline \multicolumn{6}{|l|}{ Status in 1997} \\
\hline Alive & 11289 & 3291 & 7282 & 716 & 10082 \\
\hline Dead & 5585 & 2104 & 2646 & 835 & 3356 \\
\hline Lost to follow up & 6403 & 2544 & 3512 & 347 & 9821 \\
\hline \multicolumn{6}{|l|}{ Men } \\
\hline No. & 27437 & 10059 & 15155 & 2223 & 27429 \\
\hline Mean (SD) age at diagnosis & $66.8(14.6)$ & $65.9(17.3)$ & $67.0(12.9)$ & $69.2(11.0)$ & None \\
\hline Total person-years & 78113 & 33050 & 41921 & 3142 & 118196 \\
\hline \multicolumn{6}{|l|}{ Status in 1997} \\
\hline Alive & 12084 & 3759 & 7588 & 737 & 10731 \\
\hline Dead & 8341 & 3323 & 3824 & 1194 & 5658 \\
\hline Lost to follow up & 7012 & 2977 & 3743 & 292 & 11040 \\
\hline
\end{tabular}

95\% confidence level. Prevalence and mortality rates of patients with COPD were estimated by sex, year, and age intervals using standard techniques. Log linear models were used to compare mortality distributions by sex, age strata, and disease severity. Standard survival analysis was used to construct a cohort of COPD patients and the estimation of person-years. ${ }^{11}$ Entry time in the cohort was considered as the date at which a first diagnosis of COPD was given, and the duration was defined as the time period between entry time and the time of the event or censoring, assessed in person-years by calendar time. Individuals were considered censored if no GP contact was recorded 12 months after his/her last visit. Kaplan-Maier and Cox proportional hazards estimates were obtained for stratified samples by sex, age, and severity in the survival analysis. A random sample of active (any medical contact from 1990) GPRD participants with the same sex and age structure (by five year intervals) as the COPD sample was obtained to

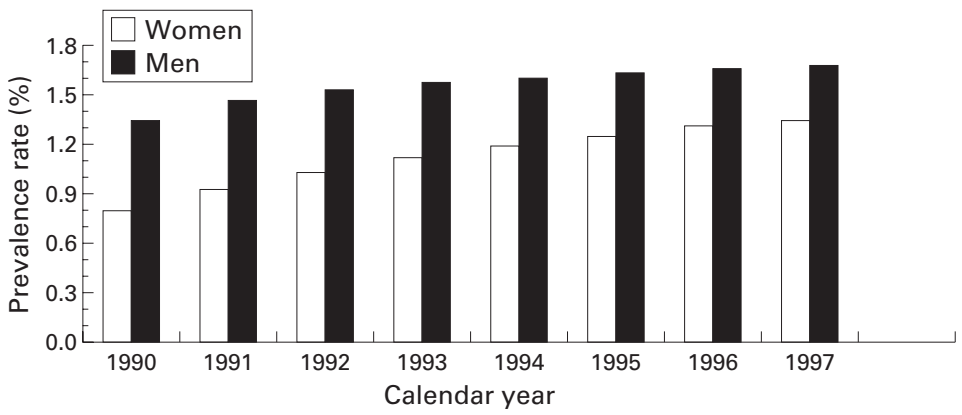

Figure 1 Prevalence (\%) of physician diagnosed COPD in the UK from 1990 to 1997 by sex.

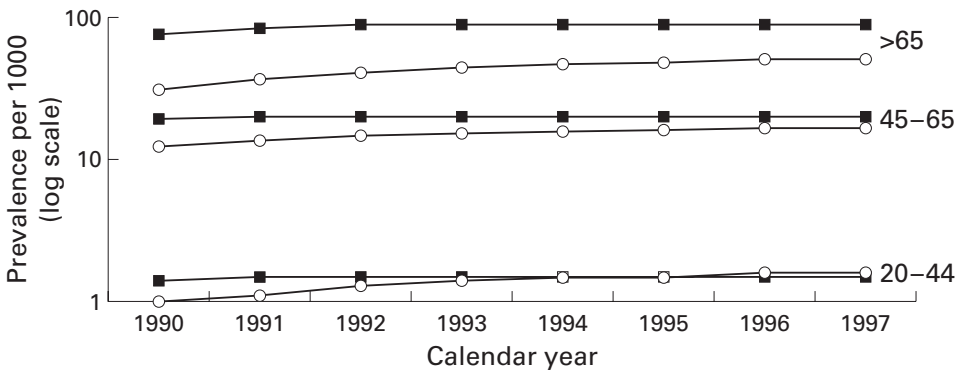

Figure 2 Age-specific and sex-specific prevalence of COPD (per thousand) in the UK from 1990 to 1997. Rates are plotted for men (प) and women (O) on a log scale for subjects aged 65+, 45-65, and 20-44 years. estimate comparisons with the general population. They are intended to be a true study base population or population frame from which one would choose all the sample - in this case patients with physician diagnosed COPD of the same age and sex. ${ }^{12}$ This GPRD control sample includes individuals registered with GPs, some of whom are healthy and some who are sick with diseases of a better/worse prognosis than COPD. All definitions, methods, and statistics were applied identically to the COPD sample and the reference non-COPD sample.

\section{Results}

From 78172 patients identified with physician diagnosed COPD 50714 were incident cases from 1 January 1990, 23277 (45.9\%) of whom were women (table 1). Total follow up was 146026 person-years stratified by severity status as: mild 59974 person-years, moderate 79929 person-years, and severe 6124 personyears. The characteristics of all patients stratified by sex are shown in table 1 . Information is also presented of the age-sex matched cohort of non-COPD participants who served as a reference population for comparison.

From 1990 to 1997 the annual prevalence rates of physician diagnosed COPD in women rose from $0.80 \%(95 \%$ CI 0.75 to 0.83$)$ to $1.36 \%$ (95\% CI 1.34 to 1.39 ), (p for trend $<0.01$ ), reaching the rate observed in men in 1990 (fig 1). The prevalence rate increased by $68.7 \%$ in women compared with $25.3 \%$ in men $(\mathrm{p}<0.05)$. COPD rates were higher in men than in women at any age interval (fig 2). While prevalence rates in men plateaued in the mid 1990 s, there was a striking increase in prevalence rates of COPD in women older than 65 years, and the rates in women aged $45-65$ years approached the rates in men in later years. Interestingly, in young adults aged $20-44$ years who, as expected, had very low rates of COPD, the prevalence in women had already surpassed the rate in men in the mid 1990s.

In table 2 all-cause mortality data are presented on the 13926 deaths observed. Mortality rates were significantly higher for COPD of any severity than for the control non-COPD population. A highly significant inverse relationship between younger age at death and increasing severity of COPD was observed. Patients with severe COPD died an 
Table 2 Number of deaths and mortality rates (per 1000 person-years) in patients with COPD and controls

\begin{tabular}{lccccc}
\hline & \multicolumn{2}{c}{ COPD } & \multicolumn{2}{c}{ Control } \\
\cline { 2 - 5 } & All & Mild & Moderate & Severe & \\
\hline Number of deaths & 13926 & 5427 & 6470 & 2029 & 9014 \\
Women, n (\%) & $5585(40.1)$ & $2104(38.7)$ & $2646(40.8)$ & $835(41.1)$ & $3356(37.2)$ \\
Mean (SD) age at death & $76.5(9.4)$ & $77.2(9.6)$ & $76.6(9.2)$ & $74.2(9.1)$ & $78.3(9.0)$ \\
Age at death, n (\%) & & & & & \\
$\quad<45$ & $38(0.2)$ & $13(0.2)$ & $18(0.3)$ & $7(0.3)$ & $14(0.2)$ \\
$\quad 45-65$ & $1591(11.4)$ & $577(10.6)$ & $713(11.0)$ & $301(14.8)$ & $652(7.2)$ \\
$\quad>65$ & $12290(88.2)$ & $4831(89.0)$ & $5739(88.7)$ & $1720(84.8)$ & $8348(92.6)$ \\
Mortality rates (per 1000 person-years) & & & & \\
Women & 72.4 & 54.3 & 69.6 & 275.7 & 30.0 \\
Men & 95.2 & 73.5 & 91.6 & 380.2 & 45.0 \\
Age & & & & 46.5 & 1.3 \\
$\quad<45$ & 5.0 & 2.3 & 6.5 & 46.5 & 11.7 \\
$\quad 45-65$ & 34.6 & 22.3 & 30.8 & 184.4 & 52.5 \\
$\quad$ All & 125.3 & 103.8 & 114.6 & 398.0 & 40.0 \\
\hline
\end{tabular}

average of three years before those with mild COPD and four years before the age and sex matched reference population $(\mathrm{p}<0.01)$. Increasing severity of COPD was associated with increased mortality. Mortality rates per 1000 person-years for non-COPD, mild, moderate and severe COPD were 30.0, 54.3, 69.6, and 275.7, respectively, in women ( $\mathrm{p}$ for trend $<0.05$ ) and 45.0, 73.5, 91.6, and 380.2 in men ( $\mathrm{p}$ for trend $<0.05$ ). Men therefore had higher mortality rates than women at each level of severity with a relative risk of 1.3 consistent at all levels. The highest mortality rate $(458.2$ per 1000 person-years) was observed in men aged over 65 years with severe COPD (data not shown). The proportion of total deaths in women increased significantly with increasing severity of COPD $(38.7 \%, 40.8 \%$, and $41.1 \%$ of deaths, respectively, for women with mild, moderate, and severe COPD; $\mathrm{p}=0.03$ ).

Finally, survival analysis (fig 3) confirmed increased mortality of patients with COPD compared with the general population even in those with mild disease, there being a greater
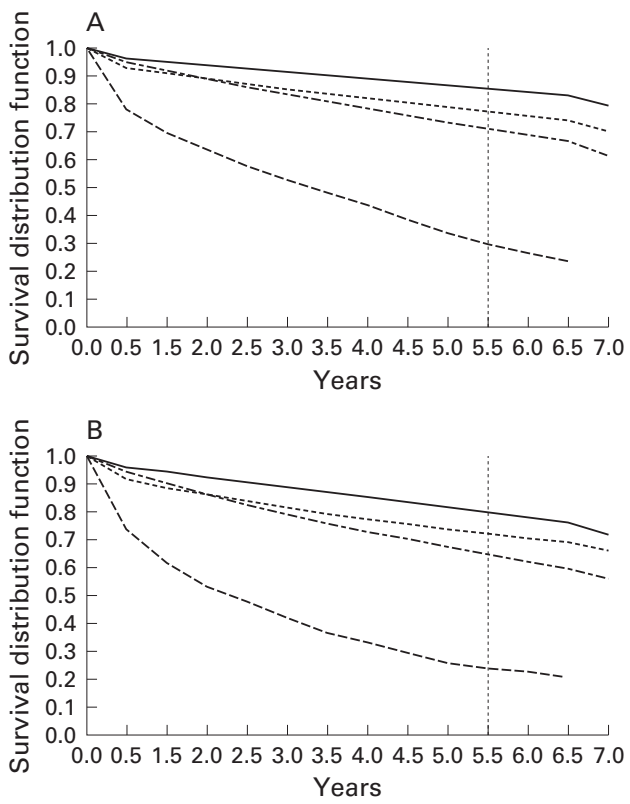

Figure 3 Survival among (A) women and $(B)$ men diagnosed with COPD by severity status and reference population . Continuous line $=$ controls $;$ dotted line $=$ mild $C O P D ;$ dashed $/$ dotted line $=$ moderate $C O P D ;$ dashed line = severe $C O P D$ mortality with increasing severity of COPD $(p<0.01)$. Women with COPD had the same pattern as men, but survival in men was worse at any given level of severity. After an arbitrary five years of follow up, survival in controls and in patients with mild, moderate, and severe COPD was $86 \%, 78 \%, 71 \%$, and $30 \%$, respectively, in women and $80 \%, 72 \%, 65 \%$, and $24 \%$, respectively, in men, all comparisons by sex within a given severity being statistically significant.

\section{Discussion}

In a retrospective cohort analysis of patients with physician diagnosed COPD made from 1990 to 1997, all-cause mortality rates were increased in patients with COPD compared with the general population. Mortality rates in patients with COPD increased with severity of the condition. For all ages and with each grade of severity of COPD the mortality rates in men were higher than in women. Currently, women account for $46 \%$ of the newly diagnosed cases of COPD in the UK population, but the trends seen in this study indicate that this proportion is rising. Before considering the significance of the present observations, two issues must be considered-limitation of the data sources and study design.

LIMITATION OF THE DATA SOURCES

A general shortcoming of using the GPRD is that it is so far underused for studies of the natural history of disease. ${ }^{13}$ Particular problems for its use in respiratory disease are the lack of valid information on respiratory function, weight, alcohol, and tobacco consumption. This prevents conventional staging of COPD severity; apart from age, the best predictor of prognosis in patients with COPD is the postbronchodilator forced expiratory volume in one second $\left(\mathrm{FEV}_{1}\right)$ expressed as a percentage of the predicted value. ${ }^{14}$ Offsetting this are the very large sample size and the extensive information of medical events and treatments which are available from practices in all parts of the UK. We acknowledge that one limitation of our validation method is that agreement for definitions of COPD and severity of COPD was investigated from information on the database and not from the patients' notes.

\section{STUDY DESIGN}

Use of a cohort from the GPRD was deemed the most appropriate design to study recent trends of COPD in the UK. The use of retrospective patient data may have led to some misclassification of COPD status. The technique we used to identify COPD could suffer from a common disadvantage of screening tools for chronic diseases - namely, that methods with good sensitivity and with reasonable specificity may detect increases in prevalence with decreases in incidence, as exemplified in cancer studies. ${ }^{15}$ Part of the reported increase in prevalence of COPD may therefore be an artefact. Because GPRD records are available only from 1987, and patient history before 1987 is incomplete, some previously prevalent patients who returned for treatment in the 
years following 1990 may be misclassified as incident COPD. There is no reason, however, to suspect that this "re-diagnosis" occurred differentially by age or sex. A number of factors might play a part in the reported increase in the prevalence of COPD including intrinsic factors of the GPRD database and study design as described above, use of a sensitive definition to screen the database for COPD cases, publicity and awareness campaigns on COPD in the lay and medical arenas, and diagnostic bias. We tried to overcome the latter by using a combination of OXMIS codes including terms with chronic bronchitis, emphysema, and COPD rather than COPD only. We reckon, however, that previously undiagnosed/ untreated patients are now seeking medical care and that smoker's cough is now more frequently diagnosed as COPD which, indeed, will increase the burden of the disease for the health system. Thus, part of the reported rise in the prevalence of COPD may be an artefact, but this effect should not differ between the sexes and therefore most of the relative increasing rates of COPD in women must be real.

Misclassification of patient mortality should also have been minimised by the use of an extended definition of death identically applied to a reference sample of non-COPD patients which, in any case, should be non-differential.

SIGNIFICANCE OF RESULTS

The overall prevalence of COPD found in this study of $0.80 \%$ in women and $1.35 \%$ in men in 1990 is comparable with other estimates, ${ }^{16}$ most of which have been extracted from the Office of National Statistics (ONS) data. ${ }^{17}{ }^{18}$ In 1995 there were 600000 patients in the UK with diagnosed COPD (overall prevalence $1 \%$, prevalence of $2 \%$ in men aged $45-65$ and $7 \%$ in men aged over 75 years). ${ }^{19}$ Although prevalence rates of COPD in subjects aged 20-44 years are obviously smaller than in the older population, these rates in young adults are of interest as they may serve as an eventual forecast for a future cohort period; it is worth noting that in 1997 the prevalence of physician diagnosed COPD in young women $(0.16 \%)$ was more frequent than in young men $(0.15 \%)$ $(\mathrm{p}=\mathrm{NS})$

The rates of COPD presented here are the lowest estimates of the population burden of COPD in the UK. Less severe COPD is commonly not diagnosed and thus these patients are not identified by the GPRD system. Indeed, physician diagnosed COPD is only the tip of the iceberg of individuals with persistently decreased lung function or a rapid decline in lung function in the general population, ${ }^{20} 21$ and it has been suggested that only $25 \%$ of COPD cases in the general population are diagnosed. ${ }^{22}$

This rising trend in prevalence of COPD in the 1990s, particularly in women, contrasts with the well established fall in COPD mortality rates observed in the UK during the last two decades. ${ }^{23}$ However, recent COPD mortality rates in the UK from 1990 to 1997 fell by $20.3 \%$ in men but increased by $12.0 \%$ in women (data extracted from several official ONS reports). In the present study we report only all-cause mortality in patients with COPD; in the USA such mortality rates are stabilising among men (except in those aged over 85 years) but are increasing in women of all ages. ${ }^{24}$ On the basis of current trends, the annual number of deaths attributable to smoking in women should exceed that for men shortly after the year 2000 in both the UK and the USA. ${ }^{23}$

The World Health Organisation indicates that adult smoking rates in the UK have continued to fall since 1980 when $42 \%$ of men and $37 \%$ of women smoked. The latest figures for smoking prevalence (age 16+) were $28 \%$ in men and $26 \%$ in women.

Susceptibility to the deleterious effects of tobacco smoking by sex deserves further consideration. This paper, and all previous literature, indicates that mortality rates from COPD are higher in men than in women. A partial explanation could be that, on average, women with COPD have less severe disease than men because women smoke less or do not inhale tobacco as frequently as men. Indeed, we observed a very consistent $30 \%$ increase in the risk of death of men versus women with COPD at any level of severity. This population finding challenges the clinical finding that women smokers have a higher prevalence of bronchial hyperresponsiveness than men, ${ }^{25}$ a risk factor for developing airflow obstruction and, for a given history of smoking, women may be more likely than men to develop obstruction. ${ }^{26}$ This indicates differences between the sexes in the presentation and pathophysiology of COPD as recognised in other diseases, ${ }^{27}$ but reasons for this need to be further investigated.

In conclusion, our results indicate that the burden of COPD is substantial among patients treated by GPs in the UK. Although men had the highest prevalence and mortality rates, women experienced the greatest increase in COPD prevalence. Because of the ageing of the population and the cumulative effect of smoking by women, the already evident burden of COPD in public health is likely to increase in the future as, indeed, Backhouse and Holland predicted 10 years ago. ${ }^{28}$ Physicians and health care providers need to prepare for this growing burden by ensuring that adequate resources are available to care for these patients. Additional education about the effects of tobacco smoking also appears to be needed to promote smoking cessation, especially in younger women.

The authors thank Alex Sexton and David Harrison for editorial assistance.

1 World Health Report. Life in the 21st century. A vision for all. Geneva: World Health Organisation, 1998.

2 Niu SR, Yang GH, Chen ZM, et al. Emerging tobacco hazards in China: 2. Early mortality results from a prospective study. BMF 1998; 317:1423-4.

3 Rawson NS, Malcolm E. Validity of the recording of ischaemic heart disease and chronic obstructive pulmonary disease in the Saskatchewan health care datafiles. Stat Med 1995;14:2627-43.

4 Sunyer J, Anto JM, McFarlane D, et al. Sex differences in mortality of people who visited emergency rooms for asthma and chronic obstructive pulmonary disease. $A \mathrm{~m} \mathcal{f}$ asthma and chronic obstructive pulm
Respir Crit Care Med 1998;158:851-6. 
5 United Kingdom of Great Britain and Northern Ireland. Tobacco or health: a global status report. Geneva: World Tobacco or health: a global status repo

6 Jick H, Jick SS, Derby LE. Validation of information recorded on general practitioner based computerised data resources in the UK. BMf 1991;302:766-8.

7 Nazareth I, King M, Haines A, et al. Accuracy of diagnosis on general practice computer system. BMF 1993;307:32-4

8 Jick H, Terris BZ, Derby LE, et al. Further validation of information recorded on a general practitioner based computerised data resource in the United Kingdom. Pharmacoepidemiol Drug Safety 1992;1:347-9.

9 Hansell A, Hollowell J, Nichols T, et al. Use of the General Practice Research Database (GPRD) for respiratory epidemiology: a comparison with the 4th Morbidity Survey in General Practice (MSGP4). Thorax 1999;54:413-9.

10 Fabbri L, Caramori G, Beghe B, et al. Chronic obstructive pulmonary disease international guidelines. Curr Opin

11 Hosmer DW, Lemeshow S, eds. Applied survival analysis: regression modelling of time to event data. Chichester: John Wiley \& Sons, 1999.

12 Miettinen OS. Theoretical epidemiology: principles of occurrence research in medicine. New York: Wiley, 1985.

13 Garcia Rodriguez LA, Perez-Gutthann S. Use of the UK General Practice Research Database for pharmacoepidemiology. Br f Clin Pharmacol 1998;45:419-25.

14 Traver GA, Cline MG, Burrows B. Predictors of mortality in chronic obstructive pulmonary disease. A 15-year follow-up study. Am Rev Respir Dis 1979;119:895-902.

15 Parkin DM, Muir CS. Comparability and quality of data. In: Parkin DM, Muir CS, Whelan SL, et al, eds. Cancer incidence in five continents. Volume VI. Lyon: IARC Scientific Publications No 120, 1992: 45-173.

16 British Lung Foundation. Chronic obstructive pulmonary disease: the key facts. British Lung Foundation, 1997.

17 Royal College of General Practitioners, Office of Population Censuses and Surveys, Department of Health. Morbidity statistics from general practice. Fourth National Study, 1991statistics from general practice. Fo
18 Royal College of General Practitioners, Office of Population Censuses and Surveys, Department of Health. Morbidity statistics from general practice. Third National Study, 1981-1982. London: HMSO, 1986.

19 Office for National Statistics. Mortality statistics: cause, England and Wales 1993 (revised) and 1994. Series DH2. No. 21. London: Government Statistical Service.

20 van den Boom G, van Schayck CP, van Mollen MP, et al. Active detection of chronic obstructive pulmonary disease and asthma in the general population. Results and economic consequences of the DIMCA program. Am $\mathcal{F}$ Respir Crit Care Med 1998;158:1730-8.

21 Crockett A. Screening older patients for obstructive airways disease. Thorax 1999;54:472-3

22 Siafakas NM, Vermeire P, Pride NB, et al. Optimal assessment and management of chronic obstructive pulmonary disease (COPD). The European Respiratory Society Task Force. Eur Respir f 1995;8:1398-420.

23 Peto R, Lopez AD, Boreham J, et al. Mortality from smoking worldwide. Br Med Bull 1996;52:12-21.

24 Mannino DM, Brown C, Giovino GA. Obstructive lung disease deaths in the United States from 1979 through 1993. An analysis using multiple-cause mortality data. Am f Respir Crit Care Med 1997;156:814-8.

25 Kanner RE, Connett JE, Altose MD, et al. Gender difference in airway hyperresponsiveness in smokers with mild COPD. The Lung Health Study. Am $\mathcal{F}$ Respir Crit Care Med 1994;150:956-61.

26 Prescott E, Bjerg AM, Andersen PK, et al. Gender difference in smoking effects on lung function and risk of ospitalization for COPD: results from a Danish longitudinal population study. Eur Respir f 1997;10:822-7.

27 Thompson PM, Wolf JL. The sexual revolution in science: what gender-based research is telling us. F Invest Med 1999; 47:106-13.

28 Backhouse A, Holland WW. Trends in mortality from chronic obstructive airways disease in the United Kingdom. Thorax 1989;44:529-32. 